\title{
Is Cardiorespiratory Disease associated with increased susceptibility of SARS-CoV-2 in Children?
}

\author{
Cassidy Du Berry ${ }^{1}$, Thomas Saunders ${ }^{1}$, Alissa McMinn ${ }^{1}$, Shidan Tosif ${ }^{1}$, Shivanthan \\ Shanthikumar ${ }^{2}$, Moya Vandeleur ${ }^{1}$, Joanne Harrison ${ }^{2}$, David Burgner ${ }^{1}$, Sarath \\ Ranganathan $^{1}$, Nigel Crawford ${ }^{1}$, and Danielle Wurzel ${ }^{1}$ \\ ${ }^{1}$ Murdoch Childrens Research Institute \\ ${ }^{2}$ The Royal Children's Hospital Melbourne
}

April 27, 2021

\begin{abstract}
Background There are limited data in paediatric populations evaluating whether chronic cardiorespiratory conditions are associated with increased risk of COVID-19. We aimed to compare the rates of chronic cardiac and respiratory disease in children testing positive (SARS-CoV-2[+]) compared to those testing negative (SARS-CoV-2[-]) at our institution. Method Prospective cohort with nested case-control study of all children tested by PCR for SARS-CoV-2 by nasopharyngeal/oropharyngeal sampling between March and October 2020. Children were identified prospectively via laboratory notification with age and sex-matching of SARS-CoV-2[+] to SARS-CoV-2[-] (1:2). Clinical data were extracted from the electronic medical record. Results In total, 179 SARS-CoV-2[+] children (44\% female, median age $3.5 \mathrm{yrs}$, range 0.1 to $19.0 \mathrm{yrs}$ ) were matched to 391 SARS-CoV-2[-] children ( $42 \%$ female, median age $3.7 \mathrm{yrs}$, range 0.1 to $18.3 \mathrm{yrs}$ ). The commonest co-morbidities showed similar frequencies in the SARS-CoV-2[+] and [-] groups: asthma ( $\mathrm{n}=9,5 \%$ vs $\mathrm{n}=17,4.4 \%, \mathrm{p}=0.71)$, congenital heart disease ( $\mathrm{n}=6,3.4 \%$ vs $\mathrm{n}=7,1.8 \%, \mathrm{p}=0.25)$ and obstructive sleep apnoea $(\mathrm{n}=4,2.2 \%$ vs $\mathrm{n}=10,2.3 \%, \mathrm{p}=0.82)$. In the SARS-CoV-2 group, the prevalence of symptomatic disease was similar amongst children with and without cardiorespiratory comorbidities $(\mathrm{n}=12,75 \%$ vs $\mathrm{n}=103,57 \%, \mathrm{p}=0.35)$ who tested positive. A high proportion of children hospitalised with SARS-CoV-2 infection had cardiac comorbidities $(23.8 \%)$. Conclusions In this single site dataset, rates of pre-existing cardiorespiratory disease were similar in SARS-CoV-2[+] and SARS-CoV-2[-] children. High rates of comorbid cardiac disease were observed amongst hospitalised children with COVID-19, warranting further research to inform public health measures and vaccine prioritisation.
\end{abstract}

\section{TITLE PAGE}

Is Cardiorespiratory Disease associated with increased susceptibility of SARS-CoV-2 in Children?

Authors: Cassidy Du Berry, BSc (Hons)*1,2; Thomas Saunders, MBBS*1,2,3; Alissa McMinn, BSc (Hons) ${ }^{1,2}$; Shidan Tosif, $\mathrm{PhD}^{1,2,3}$; Shivanthan Shanthikumar, $\mathrm{MBBS}^{1,2,3}$; Moya Vandeleur, $\mathrm{PhD}^{1,2,3}$; Joanne Harrison, MClinEd ${ }^{1,2,3}$; David Burgner, $\mathrm{PhD}^{1,2,3}$; Sarath Ranganathan $\mathrm{PhD}^{1,2,3}$; Nigel Crawford, $\mathrm{PhD}^{* 1,2,3}$; Danielle Wurzel, $\mathrm{PhD}^{* 1,2,3,4}$

*equal first/last author

${ }^{1}$ Murdoch Children's Research Institute, Infection and Immunity, Melbourne, Australia

${ }^{2}$ The Royal Children's Hospital Melbourne, Melbourne, Australia

${ }^{3}$ The University of Melbourne, Department of Paediatrics, Melbourne, Australia

${ }^{4}$ School of Population and Global Health, The University of Melbourne, Australia 


\section{Corresponding author:}

Mr. Cassidy Du Berry

Cassidy.duberry@mcri.edu.au

Department of Infection and Immunity

Murdoch Children's Research Institute

50 Flemington Road, Parkville, Victoria, 3052, Australia.

Telephone : +61393454522 Fax: +61393459154

Text word count: +1272

Key words: SARS-CoV-2, COVID-19, Child, Paediatric lung disease, Asthma, Cardiac

Declaration of interest: All authors state that they have no competing interests to declare.

\section{Abstract \\ Background}

There are limited data in paediatric populations evaluating whether chronic cardiorespiratory conditions are associated with increased risk of COVID-19. We aimed to compare the rates of chronic cardiac and respiratory disease in children testing positive (SARS-CoV-2[+]) compared to those testing negative (SARS$\mathrm{CoV}-2[-])$ at our institution.

\section{Method}

Prospective cohort with nested case-control study of all children tested by PCR for SARS-CoV-2 by nasopharyngeal/oropharyngeal sampling between March and October 2020. Children were identified prospectively via laboratory notification with age and sex-matching of SARS-CoV-2[+] to SARS-CoV-2[-] (1:2). Clinical data were extracted from the electronic medical record.

\section{Results}

In total, 179 SARS-CoV-2[+] children ( $44 \%$ female, median age $3.5 \mathrm{yrs,} \mathrm{range} 0.1$ to $19.0 \mathrm{yrs}$ ) were matched to 391 SARS-CoV-2[-] children ( $42 \%$ female, median age $3.7 \mathrm{yrs}$, range 0.1 to $18.3 \mathrm{yrs}$ ). The commonest co-morbidities showed similar frequencies in the SARS-CoV-2[+] and [-] groups: asthma $(\mathrm{n}=9,5 \%$ vs $\mathrm{n}=$ $17,4.4 \%, \mathrm{p}=0.71)$, congenital heart disease $(\mathrm{n}=6,3.4 \%$ vs $\mathrm{n}=7,1.8 \%, \mathrm{p}=0.25)$ and obstructive sleep apnoea ( $\mathrm{n}=4,2.2 \%$ vs $\mathrm{n}=10,2.3 \%, \mathrm{p}=0.82)$. In the SARS-CoV-2 group, the prevalence of symptomatic disease was similar amongst children with and without cardiorespiratory comorbidities $(\mathrm{n}=12,75 \%$ vs $\mathrm{n}$ $=103,57 \%, \mathrm{p}=0.35)$ who tested positive. A high proportion of children hospitalised with SARS-CoV-2 infection had cardiac comorbidities $(23.8 \%)$.

\section{Conclusions}

In this single site dataset, rates of pre-existing cardiorespiratory disease were similar in SARS-CoV-2[+] and SARS-CoV-2[-] children. High rates of comorbid cardiac disease were observed amongst hospitalised children with COVID-19, warranting further research to inform public health measures and vaccine prioritisation.

\section{Introduction}

There are limited data in paediatric populations evaluating whether chronic cardiac or respiratory conditions, such as congenital heart disease (CHD) and asthma, are associated with increased susceptibility to severe acute respiratory syndrome coronavirus 2 (SARS-CoV-2) infection. Understanding the comorbidity profiles of children with COVID-19 is important to inform public health measures and vaccine prioritisation. Adult data indicate that asthma and cystic fibrosis $(\mathrm{CF})$ may not affect susceptibility or outcomes to coronavirus disease 2019 (COVID-19) [1-4]. The aims of this study were to evaluate the prevalence of asthma and other 
cardiorespiratory diseases in a paediatric cohort attending a major tertiary paediatric facility (The Royal Children's Hospital (RCH) Melbourne, Australia) for SARS-CoV-2 testing. Our specific objectives were to (1) determine whether children with cardiac or respiratory comorbidities were more likely to test positive for SARS-CoV-2 than those without, and (2) if children with these pre-existing comorbidities experienced a higher rate of symptomatic infection than those without comorbidities.

\section{Methods}

This prospective cohort, within a nested case-control study, included all children consecutively tested with reverse-transcriptase polymerase chain reaction (RT-PCR) for SARS-CoV-2 from nasopharyngeal/oropharyngeal samples collected at RCH between $1^{\text {st }}$ March and 31st October 2020. SARS-CoV-2 positive (SARS-CoV-2[+]) children were age and sex-matched to consecutively tested SARS-CoV-2 negative (SARS-CoV-2[-]) controls at a ratio of 1:2. Negative controls used for matching were chosen using the following sequential criteria; (1) nearest chronological swab, (2) closest age match and (3) gender match. Symptom data and past medical history was obtained via a questionnaire completed by the child's guardian and verified against the participant's electronic medical record. Cardiac disease was defined as any previously diagnosed congenital heart disease (CHD) ranging from septal defects to cyanotic congenital heart disease. Respiratory disease was defined as previously diagnosed asthma, obstructive sleep apnoea (OSA), bronchopulmonary dysplasia (BPD), cystic fibrosis (CF), primary ciliary dyskinesia (PCD), interstitial lung disease (ILD), bronchiectasis or neuromuscular weakness. This study received ethics approval from the $\mathrm{RCH}$ Human Research Ethics Committee (HREC \#37024).

Descriptive statistics were used to summarise the cohort characteristics. Median and inter-quartile ranges were reported as data were non-parametric. Statistical analysis was performed using Stata Version 16.0 (Stata Corporation, College Station, Texas, USA). Pearson's chi-square test was used for comparison of categorical variables.

\section{Results}

In the study period, 26,819 upper respiratory tract swabs were performed at RCH, of which $179(0.68 \%)$ were SARS-CoV-2[+] (44\% female, median age $3.5 \mathrm{yrs})$. They were matched to 391 SARS-CoV-2[-] children ( $42 \%$ female, median age 3.7 years). Of the children with PCR-confirmed SARS-CoV-2 infection, 16/179 (8.9\%) had a previously documented cardiorespiratory co-morbidity. Samples from the SARS-CoV-2[+] and SARSCoV-2[-] cohorts were obtained from outpatient services $(87.6 \%$ vs $81.7 \%)$ and inpatient services $(12.4 \%$ vs $18.3 \%)$. Overall, SARS-CoV-2[+] children were no more likely than SARS-CoV-2[-] children to report a history of any cardiac or respiratory disease (figure 1). Similar rates of asthma ( $5.0 \%$ vs $4.4 \% ; \mathrm{p}=0.718$ ), CHD (3.4\% vs $1.8 \% ; \mathrm{p}=0.246)$ and OSA $(2.2 \%$ vs $2.3 \% ; \mathrm{p}=0.817)$ were observed between groups. No patient with BPD, CF PCD, ILD, bronchiectasis or neuromuscular weakness presented with SARS-CoV-2 infection. Amongst the SARS-CoV-2[+] group with a current diagnosis of asthma, two children $(\mathrm{n}=2 / 179$; $1.1 \%)$ were receiving inhaled corticosteroid therapy at the time of sampling, compared to three $(\mathrm{n}=3 / 391$, $0.8 \%$;) with asthma receiving therapy in the SARS-CoV-2[-] group. 115/179 (64.2\%) reported symptoms compared to $226 / 391(57.8 \%)$ in the SARS-CoV-2[-] group. Rates of symptomatic infection observed in the SARS-CoV-2[+] cohort were similar amongst children with and without cardiac and/or respiratory comorbidities $(60 \%$ vs $55.2 \%, \mathrm{p}=0.879)$. All asymptomatic infection in the SARS-CoV-2[+] group occurred in children undergoing contact tracing or mandatory testing of international arrivals as part of the state wide public health response.

Approximately one in ten $(\mathrm{n}=21 / 179 ; 11.7 \%)$ SARS-CoV-2 $[+]$ children were subsequently hospitalised as a result of their infection, with six $(28.6 \%)$ having a history of any cardiac and/or respiratory disease $(4$ had CHD requiring previous surgical intervention, 1 had atrial septal defect that did not require surgical intervention as well as asthma and 1 had only asthma). Two of these children were admitted for deteriorating respiratory symptoms and four were admitted for observation. No cases of surgically corrected CHD were reported among the SARS-CoV-2[-] group.

Most hospitalised children had mild respiratory symptoms and were admitted for observation, feeding sup- 
port or for reasons unrelated to SARS-CoV-2 infection. Three had severe disease requiring respiratory intervention; one had severe COVID-19 (with comorbid complex congenital heart disease), one had paediatric multi-system inflammatory syndrome temporally associated with SARS-CoV-2 infection (PIMS-TS) and one a Kawasaki's disease like presentation temporally associated with SARS-CoV-2.

\section{Discussion}

In this single centre prospective study, similar rates of cardiac and respiratory disease were observed in children infected with SARS-CoV-2 compared to uninfected.

In addition, those with a history of cardiac or respiratory disease were no more likely, than those without, to present with symptomatic infection. Our findings support those of other studies indicating that children with SARS-CoV-2 infection generally experience mild symptoms and that many have asymptomatic infection [5-8]. We have also shown that rates of asymptomatic infection are similar in children with and without cardiorespiratory comorbidities.

Interestingly, we also observed a high proportion of hospitalised children with SARS-CoV-2[+] infection had a history of cardiac disease $(23.8 \%)$. This raises the question of whether the primary reason for increased rate of hospitalisation for these children is the result of their pre-existing cardiac disease, increasing susceptibility to more severe forms of infection, or if admission was for precautionary reasons. Recent data suggests that pre-existing cardiac disease in children is associated with hospitalisation, ICU admission and mechanical ventilation [9-11]. A systematic review by Williamset al showed high rates of cardiac disease $(\mathrm{n}=11 / 48$; $23 \%$ ) in children and adolescents with COVID-19 requiring hospitalisation and mechanical ventilation [10]. Furthermore the authors also reported that cardiac disease requiring prior surgical intervention was associated with more severe forms of SARS-CoV-2 infection and higher rates of hospitalisation [10]. Cardiac disease was also shown to be associated with ICU admission in a recent European multicentre study [11]. In contrast, whilst asthma was also common in the SARS-CoV-2[+] cohort, only two (9.5\%) children who were hospitalised with COVID-19 reported a history of asthma. Similar findings have been reported in another observational study. [12]

While symptom profiles of SARS-CoV-2[+] and SARS-CoV-2[-] groups were similar (table 1) SARS-CoV$2[-]$ children were more likely, than SARS-CoV-2[+] children, to report a "runny nose", shortness of breath and/or reduced appetite. In contrast, SARS-CoV-2[+] children more commonly reported diarrhoea than the SARS-CoV-2[-] group $(6.2 \%$ vs $2 \% ; \mathrm{p}=0.011)$. Rates of cough were similar between groups and in those with and without cardiorespiratory disease (data not shown).

There are several strengths to this study. First, testing was performed in a single paediatric hospital where healthcare workers and laboratory staff adhere to strict guidelines of sample collection and processing. Second, compared to community-based settings, there was likely to be a higher proportion of children with pre-existing comorbidities, as hospital-based testing clinics generally attract patients of the hospital, we acknowledge however that this strength is simultaneously a limitation of our study as parents of children with pre-existing comorbidities may have preferentially presented to the $\mathrm{RCH}$ over their local testing centre, potentially introducing selection bias. Third, due to the low prevalence of COVID-19 in Australia, our sample size is small, however this is balanced by the high level of case ascertainment in Australia which increases the generalisability of our findings.

In conclusion, pre-existing cardiac or respiratory disease did not appear to increase susceptibility to SARSCoV-2 infection. Furthermore, children with, compared to those without, respiratory diseases had similar rates of symptomatic COVID-19. The high rates of pre-existing cardiac disease observed in hospitalised children with SARS-CoV-2 infection warrants further study.

Funding/Support: This study was supported by Murdoch Children's Research Institute, Centers of Excellence in Influenza Research and Surveillance - Cross-Center Southern Hemisphere Project, The Influenza Complications Alert Network Surveillance System, Paediatric Active Enhanced Disease Surveillance and Sentinel Travelers and Research Preparedness Platform for Emerging Infectious Disease. 


\section{References}

1. Choi, Y.J., et al., Effect of Asthma and Asthma Medication on the Prognosis of Patients with COVID-19. Eur Respir J, 2020.

2. Colombo, C., et al., Impact of COVID-19 on people with cystic fibrosis. The Lancet Respiratory Medicine, 2020. 8 (5): p. e35-e36.

3. McClenaghan, E., et al., The global impact of SARS-CoV-2 in 181 people with cystic fibrosis. Journal of Cystic Fibrosis, 2020.

4. Green, I., et al., COVID-19 Susceptibility in Bronchial Asthma. J Allergy Clin Immunol Pract, 2020.

5. Lu, X., et al., SARS-CoV-2 Infection in Children. N Engl J Med, 2020. 382 (17): p. 1663-1665.

6. Castagnoli, R., et al., Severe Acute Respiratory Syndrome Coronavirus 2 (SARS-CoV-2) Infection in Children and Adolescents: A Systematic Review. JAMA Pediatr, 2020. 174 (9): p. 882-889.

7. Patel, N.A., Pediatric COVID-19: Systematic review of the literature. Am J Otolaryngol, 2020. 41 (5): p. 102573.

8. Ludvigsson, J.F., Systematic review of COVID-19 in children shows milder cases and a better prognosis than adults. Acta Paediatr, 2020. 109 (6): p. 1088-1095.

9. Sanna, G., et al., Children's heart and COVID-19: Up-to-date evidence in the form of a systematic review. Eur J Pediatr, 2020.179 (7): p. 1079-1087.

10. Williams, N., et al., COVID-19 Severe acute respiratory syndrome coronavirus 2 (SARS-CoV-2) infection in children and adolescents: a systematic review of critically unwell children and the association with underlying comorbidities. Eur J Pediatr, 2021.180 (3): p. 689-697.

11. Götzinger, F., et al., COVID-19 in children and adolescents in Europe: a multinational, multicentre cohort study. The Lancet Child \& Adolescent Health, 2020. 4 (9): p. 653-661.

12. Chao, J.Y., et al., Clinical Characteristics and Outcomes of Hospitalized and Critically Ill Children and Adolescents with Coronavirus Disease 2019 at a Tertiary Care Medical Center in New York City. J Pediatr, 2020. 223 : p. 14-19 e2.

\section{Hosted file}

Table 1 - Study Population Characteristics.pdf available at https://authorea.com/ users/410266/articles/519714-is-cardiorespiratory-disease-associated-with-increasedsusceptibility-of-sars-cov-2-in-children

\section{Hosted file}

Table 2 - Comorbidites and Symptoms.pdf available at https://authorea.com/users/410266/ articles/519714-is-cardiorespiratory-disease-associated-with-increased-susceptibilityof-sars-cov-2-in-children 


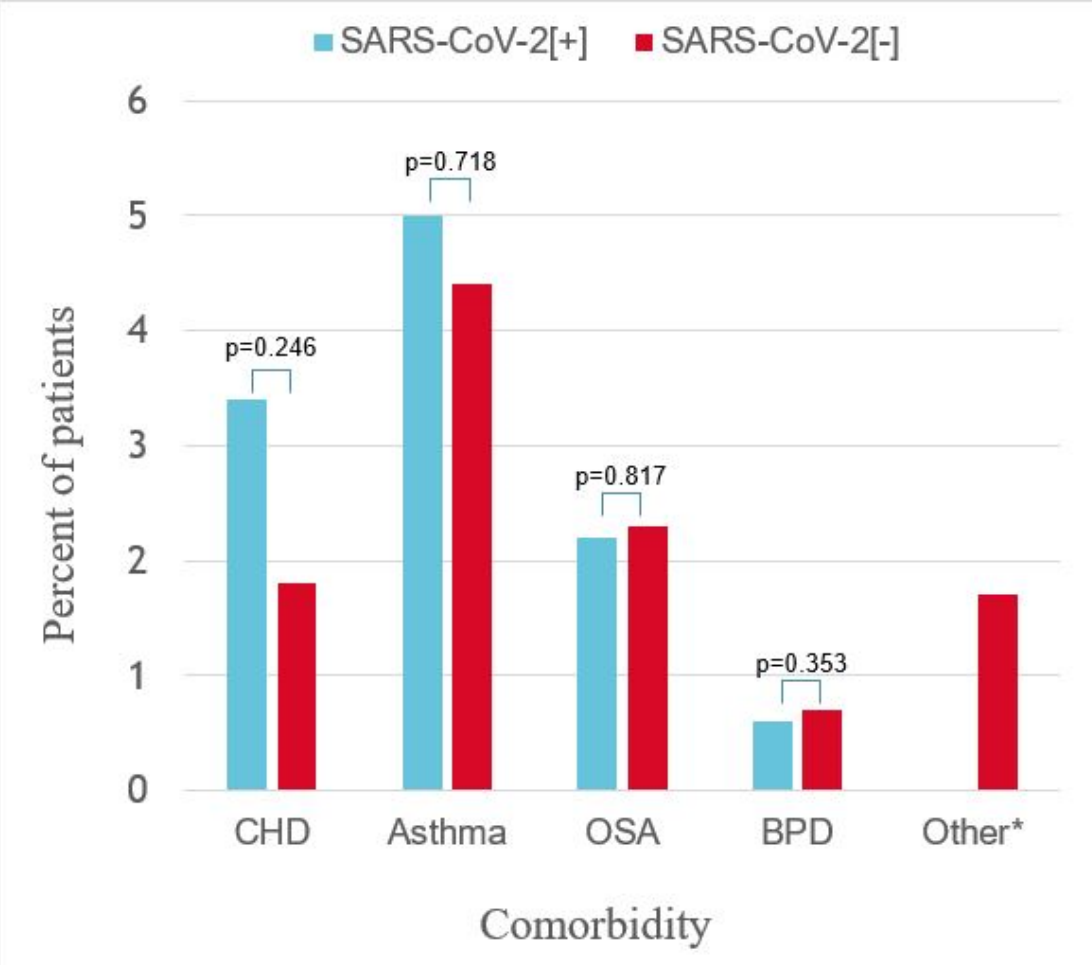

